\title{
Passive Radar Imaging of Moving Targets Using Distributed Apertures
}

\author{
Ling Wang $^{a}$ and Birsen Yazıc1 $^{b}$ \\ ${ }^{a}$ Department of Information and Communication Engineering, Nanjing University of \\ Aeronautics and Astronautics, Nanjing, Jiangsu 210016 China; \\ ${ }^{b}$ Department of Electrical, Computer and System Engineering, Rensselaer Polytechnic \\ Institute, Troy, NY 12180 USA
}

\begin{abstract}
We present a novel passive radar imaging method for moving targets using distributed apertures. We develop a passive measurement model that relates measurements at a given receiver to measurements at other receivers. We formulate the passive imaging problem as a Generalized likelihood ratio test (GLRT) for a hypothetical target located at an unknown position, moving with an unknown velocity. We design a linear discriminant functional by maximizing the signal-to-noise ratio (SNR) of the test-statistic, and use the resulting position- and velocity-resolved test-statistic to form an image of the scene of interest. We present numerical experiments to demonstrate the performance of our imaging method.
\end{abstract}

Keywords: Passive Radar, Imaging, Moving Targets, Distributed Apertures, Generalized Likelihood Ratio Test (GLRT)

\section{INTRODUCTION}

In recent years, passive radar imaging using transmitters of opportunity has emerged as an active area of research. This research effort is motivated by the increasing number of broadcasting stations, mobile phone base stations, communication and navigation satellites, particular in urban areas, as well as relatively low cost and rapid deployment of receivers. A number of passive moving target detection and imaging approaches have been developed in the literature. ${ }^{1-18}$ We present a new passive image formation method for moving targets to determine the distribution of targets in position and velocity spaces using a sparse array of receivers and non-cooperative transmitters.

We develop a new passive measurement model that relates the Doppler as well as the delay information measured at a receiver location to the delay and Doppler information measured at other receiver locations due to a hypothetical moving target in position and velocity spaces. We use the new passive measurement model to address the moving target imaging problem as a position- and velocity-resolved binary hypothesis testing, which has its root in the Generalized Likelihood Ratio Test (GLRT). ${ }^{19,20}$ The GLRT-based approach provides a particularly suitable framework for the sparse aperture arrays due to limited data available. The test-statistic produced by the hypothesis testing can be viewed as a superposition of filtered, delayed and scaled (or dilated) correlations of measurements at different receivers. We use the position- and velocity-resolved test-statistics to form an image in position and velocity spaces. We present numerical simulations to demonstrate the performance of our passive moving target imaging algorithms.

Our passive imaging method has the following advantages: (1) Unlike the existing methods that focuses on the detection of the radial velocity of moving targets ${ }^{1-16}$ using specific waveforms available in real world or target radiation, our approach determines the two- or three-dimensional velocity vector as well as two- or three-dimensional position vector of the moving target. (2) As compared to the existing passive radar detection

Further author information: (Send correspondence to B.Y.)

B.Y.: E-mail: yazici@ecse.rpi.edu, Telephone: +1(518)276 2905, Fax: +1(518)276 6261

L.W.:E-mail: wanglrpi@gmail.com

Radar Sensor Technology XV, edited by Kenneth I. Ranney, Armin W. Doerry,

Proc. of SPIE Vol. 8021, 80211F · (c) 2010 SPIE · CCC code: 0277-786X/10/\$18 .

doi: $10.1117 / 12.887452$

Proc. of SPIE Vol. $802180211 \mathrm{~F}-1$ 
methods, our approach does not require transmitters or receivers with high directivity. (3) Our approach is applicable to both cooperative and non-cooperative transmitters of opportunity.

While our treatment focuses primarily on radar imaging, our method is directly applicable to passive imaging of moving objects in seismic, acoustic and microwave imaging. Additionally, our approach can be extended to imaging of moving targets in multiple-scattering environments.

The rest our paper is organized as follows: In Section 2, we describe the models for a moving target, incident and scattered fields, and next use these to develop a passive measurement model for moving targets. In Section 3 , we use the passive measurement model developed in Section 2 to address the moving target imaging problem within the GLRT framework. In Section 4, we present numerical simulations to demonstrate the performance of the imaging method. Section 5 concludes our discussion.

\section{PASSIVE MEASUREMENT MODEL FOR MOVING TARGETS}

\subsection{Models for Moving Target, Incident and Scattered Fields}

The propagation of electromagnetic waves due to an arbitrary source distribution $s(\mathbf{x}, t)$ in a medium can be described using the scalar wave equation: ${ }^{21-23}$

$$
\left[\nabla^{2}-c^{-2}(\mathbf{x}, t) \partial_{t}^{2}\right] E(\mathbf{x}, t)=s(\mathbf{x}, t)
$$

where $c$ is the wave speed in the medium and $E$ is the electric field. Let $c_{0}$ denote the speed of light in free space, and $g$ be the Green's function satisfying

$$
\left[\nabla^{2}-c_{0}^{-2} \partial_{t}^{2}\right] g(\mathbf{x}, \mathbf{z}, t)=\delta(\mathbf{x}-\mathbf{z}) \delta(t) .
$$

The wave speed $c$ in a non-homogenous medium can be expressed in terms of the background propagation speed and the perturbation due to deviation from the background reflectivity. Let $q_{\mathbf{v}}$ denote the phase-space distribution, at time $t=0$, of scatterers moving with velocity $\mathbf{v}$. The moving scatterers in the spatial volume $d^{3} x($ at $\mathbf{x})$ give rise to ${ }^{24}$

$$
c^{-2}(t, \mathbf{x})=c_{0}^{-2}+\int q_{\mathbf{v}}(\mathbf{x}-\mathbf{v} t) d \mathbf{v} .
$$

We assume that the electromagnetic waves decay rapidly as they penetrate the ground. ${ }^{25}$ We then write $q_{\mathbf{v}}(\mathbf{x}-\mathbf{v} t)$ in terms of the two-dimensional location and two-dimensional velocity as follows:

$$
q_{\mathbf{v}}(\mathbf{x}-\mathbf{v} t)=q_{\boldsymbol{v}}(\boldsymbol{x}-\boldsymbol{v} t) \delta\left(x_{3}-h(\boldsymbol{x})\right) \delta\left(v_{3}-D h(\boldsymbol{x}) \cdot \boldsymbol{v}\right)
$$

where $\mathbf{x}=\left(\boldsymbol{x}, x_{3}\right), \boldsymbol{x} \in \mathbb{R}^{2}$ and $\mathbf{v}=\left(\boldsymbol{v}, v_{3}\right), \boldsymbol{v} \in \mathbb{R}^{2}, h: \mathbb{R}^{2} \rightarrow \mathbb{R}$ represents the ground topography and $D h(\boldsymbol{x})=\left[\begin{array}{ll}\frac{\partial h}{\partial x_{1}} & \frac{\partial h}{\partial x_{2}}\end{array}\right]$.

Let $E^{\text {sc }}$ denote the scattered field due to the moving perturbation $q_{\boldsymbol{v}}$. Then, under the Born approximation ${ }^{26}$ and the slow-mover assumption, i.e., the speed of the target, $|\boldsymbol{v}|$, is much slower than the speed of light, the scattered field is modeled as

$$
E^{\mathrm{sc}}(\mathbf{x}, t)=\int g\left(\mathbf{x}, \boldsymbol{y}, t-\mu_{\boldsymbol{y}, \boldsymbol{v}, \mathbf{x}} \tau\right) q_{\boldsymbol{v}}(\boldsymbol{y}) \partial_{\tau}^{2} \tilde{E}^{\mathrm{in}}(\boldsymbol{y}, \tau) d \tau d \boldsymbol{y} d \boldsymbol{v}
$$

where

$$
\tilde{E}^{\mathrm{in}}(\boldsymbol{y}, \tau)=\sum_{q=1}^{M} \int g\left(\mathbf{y}, \mathbf{z}_{q}, \tilde{\mu}_{\boldsymbol{y}, \boldsymbol{v}, \mathbf{z}_{q}} \tau-\tau^{\prime}\right) p_{q}\left(\tau^{\prime}+T_{\mathbf{z}_{q}}\right) d \tau^{\prime}
$$

and

$$
\begin{gathered}
\mu_{\boldsymbol{y}, \boldsymbol{v}, \mathbf{x}}=1+\frac{\widehat{\mathbf{y - \mathbf { x }}} \cdot \mathbf{v}}{c_{0}}, \\
\tilde{\mu}_{\boldsymbol{y}, \boldsymbol{v}, \mathbf{z}_{q}}=1-\frac{\widehat{\mathbf{y - \mathbf { z } _ { q }} \cdot \mathbf{v}}}{c_{0}} .
\end{gathered}
$$


In (5), $g(\mathbf{x}, \boldsymbol{y}, t)$ is defined as the 3D Green's function of the background environment that is equal to $g(\mathbf{x},(\boldsymbol{y}, h(\boldsymbol{y})), t)$. In $(6), g(\boldsymbol{y}, \mathbf{z}, t)$ is the 3D Green's function defined as $g((\boldsymbol{y}, h(\boldsymbol{y})), \mathbf{z}, t)$.

Note that (6) represents the incident field observed by a moving target with velocity $\boldsymbol{v}$ due to transmitters located at $\mathbf{z}_{q}, q=1, \cdots, M$ transmitting waveforms $\hat{p}_{q}$ starting at time $t=T_{\mathbf{z}_{q}}$, which accounts for the Doppler scaling effect induced by the movement of the target.

The scale factor, $\tilde{\mu}_{\boldsymbol{y}, \boldsymbol{v}, \mathbf{z}_{q}}$ in (8) accounts for the Doppler scaling effect induced by the movement of the target on the field at $\boldsymbol{y}$ due to a source located at $\mathbf{z}_{q}$. We refer to $\tilde{\mu}_{\boldsymbol{y}, \boldsymbol{v}, \mathbf{z}_{q}}$ as the Doppler-scale-factor observed by the moving target located at $\boldsymbol{y}$ moving with velocity $\boldsymbol{v}$ due to a waveform transmitted from $\mathbf{z}_{q}$. The scale factor, $\mu_{\boldsymbol{y}, \boldsymbol{v}, \mathbf{x}}$ in (7) accounts for the Doppler scaling effect observed at the receiver location $\mathbf{x}$ due to a moving target with velocity $\boldsymbol{v}$. We refer to $\mu_{\boldsymbol{y}, \boldsymbol{v}, \mathbf{x}}$ as the Doppler-scale-factor observed at location $\mathbf{x}$ due to a moving target with velocity $\boldsymbol{v}$ at location $\boldsymbol{y}$. In the discussion that follows, we focus on the slow-mover case.

Using (5), in Fourier domain the measurement at the receiver located at $\mathbf{x}_{0}$ contaminated with additive thermal noise $n(t)$ can be modeled as

$$
\hat{m}(\omega)=\int \hat{g}\left(\mathbf{x}_{0}, \boldsymbol{y}, \omega\right) q_{\boldsymbol{v}}(\boldsymbol{y}) \mu_{\boldsymbol{y}, \boldsymbol{v}, \mathbf{x}_{0}}^{2} \omega^{2} \hat{\tilde{E}}^{\mathrm{in}}\left(\boldsymbol{y}, \mu_{\boldsymbol{y}, \boldsymbol{v}, \mathbf{x}_{0}} \omega\right) d \boldsymbol{y} d \boldsymbol{v}+\hat{n}(\omega)
$$

where we assume that $n$ is zero-mean with finite second-order statistics, and

$$
\hat{\tilde{E}}^{\text {in }}(\boldsymbol{y}, \omega)=-\sum_{q=1}^{M} \frac{1}{\tilde{\mu}_{\boldsymbol{y}, \boldsymbol{v}, \mathbf{z}_{q}}^{3}} \hat{g}\left(\mathbf{y}, \mathbf{z}, \frac{\omega}{\tilde{\mu}_{\boldsymbol{y}, \boldsymbol{v}, \mathbf{z}_{q}}}\right) \hat{p}_{q}\left(\frac{\omega}{\tilde{\mu}_{\boldsymbol{y}, \boldsymbol{v}, \mathbf{z}_{q}}}\right) \mathrm{e}^{\mathrm{i} \frac{\omega}{\tilde{\mu}_{\boldsymbol{y}, \boldsymbol{v}, \mathbf{z}_{q}}} T_{\mathbf{z}_{q}}} .
$$

Note that since the waveform $\hat{p}$ is typically band-limited, we restrict our analysis to some range of $\omega$.

\subsection{Passive Measurement Model}

In the analysis that follows, we consider $N$ receivers located at $\mathbf{x}_{i}, i=1, \cdots, N$ and $M$ transmitters located at $\mathbf{z}_{q}, q=1, \cdots, M$. The receivers and transmitters are arbitrarily located with several hundred wavelengths apart with known receiver locations, but unknown transmitter locations.

For the rest of our development we assume that the phase-space distribution $q_{\boldsymbol{v}}(\boldsymbol{y})$ is deterministic. This assumption allows us to simplify the analysis and distill the important aspects of our imaging theory. The results can be easily extended to statistical targets embedded in clutter.

For non-cooperative passive detection and imaging applications, the information on the transmitted waveforms and the location of the transmitters is not available. Since the measurements at all receivers are due to the same incident field $\tilde{E}^{\text {in }}$, target velocity and phase space distribution, we express the measurement, $\hat{m}_{i}$, at the $i^{\text {th }}$ receiver in terms of the measurement, $\hat{m}_{j}$, at the $j^{\text {th }}$ receiver by back-propagating $\hat{m}_{j}$ measured at $\mathbf{x}_{j}$ to a hypothetical target location with a hypothetical velocity via the back-propagation operator; and then forward propagating the resulting field to $\mathbf{x}_{i}$ via the forward-propagation operator.

In many applications, the moving target model can be simplified to a point scatterer moving at a constant velocity, i.e.,

$$
q_{\boldsymbol{v}}(\boldsymbol{y})=\rho \delta\left(\boldsymbol{y}-\boldsymbol{y}_{0}\right) \delta\left(\boldsymbol{v}-\boldsymbol{v}_{0}\right)
$$

where $\rho$ is the reflectivity of the point target located at $\boldsymbol{y}_{0}$, at time $t=0$, moving with velocity $\boldsymbol{v}_{0}$.

Thus, for a point target moving at a constant velocity, we express the forward-propagation operator as

$$
\mathcal{P}_{\boldsymbol{y}_{0}, \boldsymbol{v}_{0}, i}[u](\omega)=\hat{g}\left(\mathbf{x}_{i}, \boldsymbol{y}_{0}, \omega\right) \mu_{\boldsymbol{y}_{0}, \boldsymbol{v}_{0}, i} u\left(\mu_{\boldsymbol{y}_{0}, \boldsymbol{v}_{0}, i} \omega\right)
$$

where $\mathcal{P}_{\boldsymbol{y}, \boldsymbol{v}, i}$ denotes the forward operator with respect to the $i^{\text {th }}$ receiver,

$$
u(\boldsymbol{y}, \boldsymbol{v}, \omega)=q_{\boldsymbol{v}}(\boldsymbol{y}) \omega^{2} \hat{\tilde{E}}^{\text {in }}(\boldsymbol{y}, \omega),
$$


$\hat{g}\left(\mathbf{x}_{i}, \boldsymbol{y}_{0}, \omega\right)$ is given by

$$
\hat{g}\left(\mathbf{x}_{i}, \boldsymbol{y}_{0}, \omega\right)=\frac{\mathrm{e}^{-\mathrm{i} \frac{\omega}{c_{0}}\left|\mathbf{x}_{i}-\mathbf{y}_{0}\right|}}{4 \pi\left|\mathbf{x}_{i}-\mathbf{y}_{0}\right|}
$$

and $\mu_{\boldsymbol{y}_{0}, \boldsymbol{v}_{0}, i}$ is given by (7). Accordingly, the back-propagation operator can be expressed as

$$
\mathcal{P}_{\boldsymbol{y}_{0}, \boldsymbol{v}_{0}, i}^{-1}\left[\hat{m}_{i}\right](\omega)=\frac{1}{\mu_{\boldsymbol{y}_{0}, \boldsymbol{v}_{0}, i}} \hat{m}_{i}\left(\frac{\omega}{\mu_{\boldsymbol{y}_{0}, \boldsymbol{v}_{0}, i}}\right) \frac{1}{\hat{g}\left(\mathbf{x}_{i}, \boldsymbol{y}_{0}, \frac{\omega}{\mu_{\boldsymbol{y}_{0}, \boldsymbol{v}_{0}, i}}\right)} .
$$

where $\mathcal{P}_{\boldsymbol{y}, \boldsymbol{v}, i}^{-1}$ denotes the back-propagation operator, which is defined as the inverse of $\mathcal{P}_{\boldsymbol{y}, \boldsymbol{v}, i}$.

Using (12) and (15), for a moving point target, the full expression for the passive measurement model becomes

$$
\begin{aligned}
\hat{m}_{i}(\omega) & =\mathcal{P}_{\boldsymbol{y}_{0}, \boldsymbol{v}_{0}, i} \mathcal{P}_{\boldsymbol{y}_{0}, \boldsymbol{v}_{0}, j}^{-1} \hat{m}_{j}(\omega)+\hat{n}_{i}(\omega) \\
& =\gamma_{\boldsymbol{y}_{0}, \boldsymbol{v}_{0}, i j} \hat{m}_{j}\left(\gamma_{\boldsymbol{y}_{0}, \boldsymbol{v}_{0}, i j} \omega\right) \frac{\hat{g}\left(\mathbf{x}_{i}, \boldsymbol{y}_{0}, \omega\right)}{\hat{g}\left(\mathbf{x}_{j}, \boldsymbol{y}_{0}, \gamma_{\boldsymbol{y}_{0}, \boldsymbol{v}_{0}, i j} \omega\right)}+\hat{n}_{i}(\omega)
\end{aligned}
$$

where $n_{i}$ denotes the noise at the $i^{\text {th }}$ receiver, $\gamma_{\boldsymbol{y}, \boldsymbol{v}, i j}$ is the ratio of the Doppler-scale-factors with respect to the $i^{\text {th }}$ and $j^{\text {th }}$ receivers given by

$$
\gamma_{\boldsymbol{y}_{0}, \boldsymbol{v}_{0}, i j}:=\frac{\mu_{\boldsymbol{y}_{0}, \boldsymbol{v}_{0}, i}}{\mu_{\boldsymbol{y}_{0}, \boldsymbol{v}_{0}, j}}=\frac{1+\widehat{\mathbf{y}_{0}-\mathbf{x}_{i}} \cdot \mathbf{v}_{0} / c_{0}}{1+\widehat{\mathbf{y}_{0}-\mathbf{x}_{j}} \cdot \mathbf{v}_{0} / c_{0}}
$$

with $\mathbf{y}_{0}=\left(\boldsymbol{y}_{0}, h\left(\boldsymbol{y}_{0}\right)\right)$ and $\mathbf{v}_{0}=\left(\boldsymbol{v}_{0}, D h\left(\boldsymbol{y}_{0}\right) \cdot \boldsymbol{v}_{0}\right)$. Note that $\gamma_{\boldsymbol{y}, \boldsymbol{v}, i j}$ is the Doppler-hitchhiker-scale-factor defined in. $^{27}$ Due to the static nature of the receivers in the present work, we refer to $\gamma_{\boldsymbol{y}, \boldsymbol{v}, i j}$ as the passive-Dopplerscale-factor.

Substituting (14) into (17), we obtain

$$
\hat{m}_{i}(\omega)=\frac{\left|\mathbf{y}_{0}-\mathbf{x}_{j}\right|}{\left|\mathbf{y}_{0}-\mathbf{x}_{i}\right|} \gamma_{\boldsymbol{y}_{0}, \boldsymbol{v}_{0}, i j} \mathrm{e}^{-\mathrm{i} k\left(\left|\mathbf{y}_{0}-\mathbf{x}_{i}\right|-\gamma_{\boldsymbol{y}_{0}, \boldsymbol{v}_{0}, i j}\left|\mathbf{y}_{0}-\mathbf{x}_{j}\right|\right)} \hat{m}_{j}\left(\gamma_{\boldsymbol{y}_{0}, \boldsymbol{v}_{0}, i j} \omega\right)+\hat{n}_{i}(\omega)
$$

where $k=\omega / c_{0}$.

For $N$ receivers located at $\mathbf{x}_{1}, \ldots \mathbf{x}_{N}$, a vector measurement model can be formed by taking one of the receivers as a reference. Without loss of generality, we take the $j^{\text {th }}$ receiver as a reference and form the following measurement vector:

$$
\mathbf{m}=\left[\begin{array}{llll}
\hat{m}_{1} & \hat{m}_{2} & \cdots & \hat{m}_{N}
\end{array}\right]^{T}
$$

Similarly, we vectorize the "reference measurements" and the noise as follows:

$$
\begin{aligned}
\mathbf{m}_{\mathbf{r}} & =\left[\begin{array}{llll}
\hat{m}_{j} & \hat{m}_{j} & \cdots & \hat{m}_{j}
\end{array}\right]^{T} \\
\mathbf{n} & =\left[\begin{array}{llll}
\hat{n}_{1} & \hat{n}_{2} & \cdots & \hat{n}_{N}
\end{array}\right]^{T}
\end{aligned}
$$

where $\hat{n}_{i}, i \neq j$ is the additive thermal noise at the $i^{\text {th }}$ receiver. Note that $\mathbf{m}, \mathbf{m}_{\mathbf{r}}$ and $\mathbf{n}$ are all $(N-1)$ dimensional vectors.

The composition of the back-propagation and forward-propagation operators can be represented as a diagonal matrix given by

$$
\mathbf{P}_{\boldsymbol{y}, \boldsymbol{v}}=\operatorname{diag}\left[\begin{array}{llll}
\mathcal{P}_{\boldsymbol{y}, \boldsymbol{v}, 1} \mathcal{P}_{\boldsymbol{y}, \boldsymbol{v}, j}^{-1} & \mathcal{P}_{\boldsymbol{y}, \boldsymbol{v}, 2} \mathcal{P}_{\boldsymbol{y}, \boldsymbol{v}, j}^{-1} & \cdots & \mathcal{P}_{\boldsymbol{y}, \boldsymbol{v}, N} \mathcal{P}_{\boldsymbol{y}, \boldsymbol{v}, j}^{-1}
\end{array}\right]
$$

where $i \neq j$ and $\mathbf{P}_{\boldsymbol{y}, \boldsymbol{v}}$ is $(N-1) \times(N-1)$.

Using (16), (20)-(23), we form a vectorized passive measurement model as follows:

$$
\mathbf{m}(\omega)=\mathbf{P}_{\boldsymbol{y}, \boldsymbol{v}} \mathbf{m}_{\mathbf{r}}(\omega)+\mathbf{n}(\omega)
$$

for some range of $\omega$. Note that in (24), all operations are understood to be elementwise. 


\section{IMAGING OF MOVING TARGETS AS A POSITION- AND VELOCITY-RESOLVED BINARY HYPOTHESIS TEST}

We address the imaging formation as a Generalized Likelihood Ratio Test (GLRT) ${ }^{19,20}$ for unknown target position and velocity using the passive moving target imaging model introduced in Section (2.2).

We determine a test-statistic by maximizing the Signal-to-Noise Ratio (SNR) of the test-statistic while constraining the associated discriminant functional to be linear. The image is then formed in the $(\boldsymbol{y}, \boldsymbol{v})$ domain with the position- and velocity-resolved test-statistic where the location, possibly the shapes of the targets; and their velocities can be identified by thresholding the resulting four-dimensional test-statistic image.

The passive moving target imaging problem can be formulated as a test of the following position- and velocityresolved binary hypothesis:

$$
\begin{array}{ll}
\mathcal{H}_{0}: & \mathbf{m}=\mathbf{n} \\
\mathcal{H}_{1}: & \mathbf{m}=\mathbf{P}_{\boldsymbol{y}, \boldsymbol{v}} \mathbf{m}_{\mathbf{r}}+\mathbf{n}
\end{array}
$$

where $\mathbf{P}_{\boldsymbol{y}, \boldsymbol{v}}, \mathbf{m}_{\mathbf{r}}, \mathbf{m}$ and $\mathbf{n}$ are as defined in (20)-(24).

The null hypothesis states that the measurement is due to noise whereas the alternative hypothesis states that the measurement is due to a target located at $\boldsymbol{y}$ moving with velocity $\boldsymbol{v}$.

Using (21), (22), (25), we obtain

$$
\begin{aligned}
\mathrm{E}\left[\mathbf{m} \mid \mathcal{H}_{0}\right] & =\mathbf{0} \\
\operatorname{Cov}\left[\mathbf{m} \mid \mathcal{H}_{0}\right] & =\mathbf{R}_{\mathbf{n}}=: \mathbf{R}_{0} \\
\mathrm{E}\left[\mathbf{m} \mid \mathcal{H}_{1}\right] & =\mathbf{P}_{\boldsymbol{y}, \boldsymbol{v}} \mathrm{E}\left[\mathbf{m}_{\mathbf{r}} \mid \mathcal{H}_{1}\right]=\mathbf{P}_{\boldsymbol{y}, \boldsymbol{v}} \overline{\mathbf{m}}_{\mathbf{r}} \\
\operatorname{Cov}\left[\mathbf{m} \mid \mathcal{H}_{1}\right] & =\mathbf{P}_{\boldsymbol{y}, \boldsymbol{v}} \mathbf{R}_{\mathbf{n}_{\mathbf{r}}} \mathbf{P}_{\boldsymbol{y}, \boldsymbol{v}}^{H}+\mathbf{R}_{\mathbf{n}}=: \mathbf{R}_{1}
\end{aligned}
$$

where E denotes the expectation operator and Cov denotes the covariance operator; $\overline{\mathbf{m}}_{\mathbf{r}}$ denotes $\mathrm{E}\left[\mathbf{m}_{\mathbf{r}} \mid \mathcal{H}_{1}\right] ; \mathbf{P}_{\boldsymbol{y}, \boldsymbol{v}}^{H}$ denotes the Hermitian transpose of $\mathbf{P}_{\boldsymbol{y}, \boldsymbol{v}} ; \mathbf{R}_{\mathbf{n}}$ denotes the autocovariance of the noise vector $\mathbf{n}$, i.e., $\mathbf{R}_{\mathbf{n}}\left(\omega, \omega^{\prime}\right)=$ $\mathrm{E}\left[\mathbf{n}(\omega) \mathbf{n}^{H}\left(\omega^{\prime}\right)\right]$ and $\mathbf{R}_{\mathbf{n}_{\mathbf{r}}}$ denotes the autocovariance of the noise vector $\mathbf{n}_{\mathbf{r}}:=\left[\hat{n}_{j}, \hat{n}_{j}, \cdots, \hat{n}_{j}\right]$, i.e., $\mathbf{R}_{\mathbf{n}_{\mathbf{r}}}\left(\omega, \omega^{\prime}\right)=$ $\mathrm{E}\left[\mathbf{n}_{\mathbf{r}}(\omega) \mathbf{n}_{\mathbf{r}}^{H}\left(\omega^{\prime}\right)\right]$.

The linear discriminant functional involved in our problem has the form

$$
\lambda=\langle\mathbf{m}, \mathbf{w}\rangle:=\int \mathbf{w}^{H} \mathbf{m} d \omega=\sum_{i, i \neq j} \int w_{i}^{*}(\omega) \hat{m}_{i}(\omega) d \omega
$$

where $\lambda$ denotes the the output of the discriminant functional, which we call the test-statistic, and $\mathbf{w}$ is a template given by

$$
\mathbf{w}=\left[\begin{array}{llll}
w_{1} & w_{2} & \cdots & w_{N}
\end{array}\right]^{T} .
$$

We determine the template by maximizing the SNR of $\lambda$.

Under the assumption that the noise at different receivers are uncorrelated and wide-sense stationary, the resulting optimal template is

$$
\mathbf{w}_{\mathrm{opt}}=\overline{\mathbf{S}}^{-1}(\omega) \mathbf{P}_{\boldsymbol{y}, \boldsymbol{v}}(\omega) \overline{\mathbf{m}}_{\mathbf{r}}(\omega)
$$

where $\overline{\mathbf{S}}^{-1}$ is the inverse of $\overline{\mathbf{S}}=1 / 2\left(\mathbf{R}_{1}+\mathbf{R}_{0}\right)$. One can show that $\overline{\mathbf{S}}^{-1}$ can be approximated by a diagonal matrix. We denote diagonal elements of $\overline{\mathbf{S}}^{-1}$ by $\bar{S}_{i}^{-1}(\omega), i=1, \cdots, N$ and $i \neq j$, which is a function of the power spectral density function of the noise, $S_{n}^{i}(\omega)$ and the kernel of $\mathbf{P}_{\boldsymbol{y}, \boldsymbol{v}}$.

For a moving point target located at $\boldsymbol{y}$, moving with a hypothetical velocity $\boldsymbol{v}$, each component of the optimal template becomes

$$
w_{i}=\bar{S}_{i}^{-1}(\omega) \frac{\left|\mathbf{y}-\mathbf{x}_{j}\right|}{\left|\mathbf{y}-\mathbf{x}_{i}\right|} \gamma_{\boldsymbol{y}, \boldsymbol{v}, i j} \mathrm{e}^{-\mathrm{i} k\left(\left|\mathbf{y}-\mathbf{x}_{i}\right|-\gamma_{\boldsymbol{y}, \boldsymbol{v}, i j}\left|\mathbf{y}-\mathbf{x}_{j}\right|\right)} \mathrm{E}\left[\hat{m}_{j}\left(\gamma_{\boldsymbol{y}, \boldsymbol{v}, i j} \omega\right)\right]
$$

where $i=1, \cdots, N$ and $i \neq j$ and $\gamma_{\boldsymbol{y}, \boldsymbol{v}, i j}$ is given by (18). 
The first term in (33) is a prewhitening filter due to colored noise, the second term involves scaling due to geometrical spreading factors and the passive-Doppler-scale-factor, the third term involves delay due to the path difference between the two receivers and a temporal dilation due to the passive-Doppler-scale-factor, and the last term involves averaged dilated reference measurement.

Thus, the output of the linear discriminant functional can be viewed as a summation of the correlations between the filtered, delayed, scaled (or dilated) replica of the reference measurement $m_{j}$ and the measurement $m_{i}, i \neq j$, which is given by

$$
\lambda(\boldsymbol{y}, \boldsymbol{v})=\sum_{i \neq j} \int \frac{\left|\mathbf{y}-\mathbf{x}_{j}\right|}{\left|\mathbf{y}-\mathbf{x}_{i}\right|} \mathrm{E}\left[m_{j}^{\prime}\left(\frac{t-\left|\mathbf{y}-\mathbf{x}_{i}\right| / c_{0}}{\gamma_{\boldsymbol{y}, \boldsymbol{v}, i j}}+\frac{\left|\mathbf{y}-\mathbf{x}_{j}\right|}{c_{0}}\right)\right] m_{i}^{*}(t) d t
$$

where $m_{j}^{\prime}(t)$ is the filtered version of $m_{j}(t)$ with the filtering given by $\bar{S}_{i}^{-1}(\omega)$.

Note that for stationary targets, the passive-Doppler-scaling factor, $\gamma_{\boldsymbol{y}, \boldsymbol{v}, i j}$ becomes 1 , and the test-statistic in (34) reduces to the one in passive imaging of stationary targts using distributed apertures in free-space. ${ }^{28}$

\section{NUMERICAL SIMULATIONS}

We conducted numerical simulations to demonstrate the performance of our passive imaging method using a moving point target model.

We reconstructed the four-dimensional test-statistic image in $(\boldsymbol{y}, \boldsymbol{v})\left(\right.$ or $\left.\left(y_{1}, y_{2}, v_{1}, v_{2}\right)\right)$ coordinates. However, in order to facilitate visualization and performance evaluation, we generated three two-dimensional images from the original four dimensional image: The fist image, which we refer to as the peak-value image, was generated by choosing the maximum value of the four-dimensional image for each velocity $\left(v_{1}, v_{2}\right)$. We then, choose the maximum value, $\tilde{\boldsymbol{v}}$, of the peak-value image as the estimate of the velocity. The second image, which we refer to as the position-image, is the cross-section of the four-dimensional image, for $\boldsymbol{v}=\tilde{\boldsymbol{v}}$. We take the maximum value, $\tilde{\boldsymbol{y}}$, of the position-image as the estimate of the target's position. The third image, which we refer to as the velocity-image, is the cross-section of the four-dimensional image, for $\boldsymbol{y}=\tilde{\boldsymbol{y}}$. We performed the performance evaluation using the position- and velocity-images.

\subsection{Simulation Parameters}

We considered a scene of size $[0,3 \mathrm{e} 3] \times[0,3 \mathrm{e} 3] \mathrm{m}^{2}$ with flat topography. We discretized the scene into $201 \times 201$ pixels where $[0,0,0] \mathrm{m}$ and $[3 \mathrm{e} 3,3 \mathrm{e} 3,0] \mathrm{m}$ correspond to the pixels $(1,1)$ and $(201,201)$, respectively. We assumed that the target velocity is in the range of $[-20,20] \times[-20,20] \mathrm{m} / \mathrm{s}$. We discretized the velocity plane into $401 \times 401$ pixels where $[-20,-20,0] \mathrm{m}$ and $[20,20,0] \mathrm{m}$ correspond to the pixels $(1,1)$ and $(401,401)$, respectively.

The point target with unit reflectivity was assumed to be located at $\left[\begin{array}{lll}2.5 \mathrm{e} 3 & 2 \mathrm{e} 3 & 0\end{array}\right]^{T}$ m moving with velocity $[-10,15] \mathrm{m} / \mathrm{s}$.

Taking into account the waveforms available in practice as illuminators of opportunity, we used the type of waveforms having high Doppler resolution and relatively poor range resolution in our simulations. Examples for such waveforms include Frequency Modulated (FM) radio and television signals, waveforms used by Global System for Mobile Communication (GSM), etc. We simulated the high-Doppler resolution waveform as a singlefrequency continuous-wave $(\mathrm{CW})$ with $4 \mathrm{GHz}$ carrier frequency and $0.1 \mathrm{~s}$ duration in our experiments. Such a waveform provides a radial velocity resolution of about $0.375 \mathrm{~m} / \mathrm{s}$ in monostatic operations.

We performed the image reconstruction considering the following number of receivers and transmitters: (a)a single transmitter and 3 receivers; (b) a single transmitter and 5 receivers; (c) two transmitter and 5 receivers. For the two-transmitter case, we assumed that both transmitters are transmitting the same waveform.

Both the transmitter and receivers were assumed to be on the same $z$-plane, $z=6$. The receivers were assumed to lie on the $\mathrm{x}$-axis, equidistant form each other in the range of $[0,3 \mathrm{e} 3]^{T} \mathrm{~m}$ for the three-receiver and five-receiver cases.. The single transmitter was assumed to be located at $[1.5 \mathrm{e} 3,0,6]^{T} \mathrm{~m}$. For two-transmitter case, the transmitters were located at $\left[\begin{array}{lll}0.5 e 3 & 0 & 6\end{array}\right]^{T} \mathrm{~m}$ and $\left[\begin{array}{lll}1.5 e 3 & 0 & 6\end{array}\right]^{T} \mathrm{~m}$, all in meters. .

In all the experiments, we simulated the thermal noise as an additive white Gaussian process. 


\subsection{Results}

We used the high-Doppler resolution waveform described in Section 4.1 in the simulation experiment. The reconstructed images of the moving point target using a single transmitter and 3 and 5 receivers are shown in Fig. 1, Fig. 2, respectively.

Fig. 1(a) and Fig. 2(a) show the peak-value images formed using 3 and 5 receivers, respectively. The maximum value of the peak-value image, which corresponds to the estimated velocity, $\tilde{\boldsymbol{v}}$, is indicated by a circle. Fig. 1(b) and Fig. 2(b) show the corresponding position-images (cross-section of the four-dimensional image) when $\boldsymbol{v}=\tilde{\boldsymbol{v}}$. The maximum value of the position-image, which corresponds to the estimated position, $\tilde{\boldsymbol{y}}$, is indicated by a red circle. For ease of comparison, the true position of the target is indicated by a solid red dot. Fig. 1(c) and Fig. 2(c) show the corresponding velocity-images (cross-section of the four-dimensional image) when $\boldsymbol{y}=\tilde{\boldsymbol{y}}$. The maximum value of the velocity-image, i.e., the estimated velocity is indicated by a blue circle. The true velocity is indicated by a solid blue dot. Note that if the estimated value is almost equal to the true value, only the marker for true value is shown.

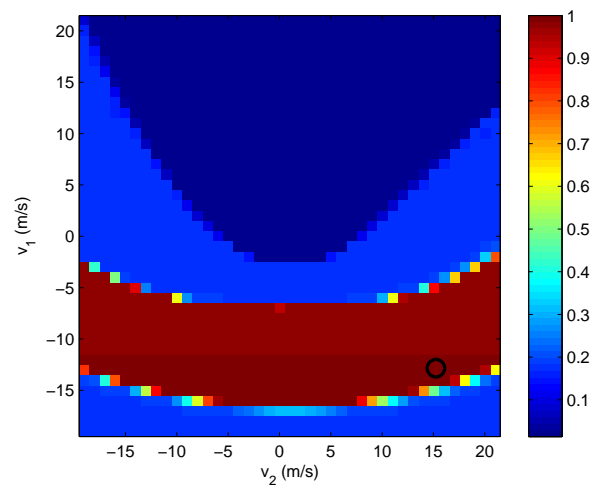

(a)

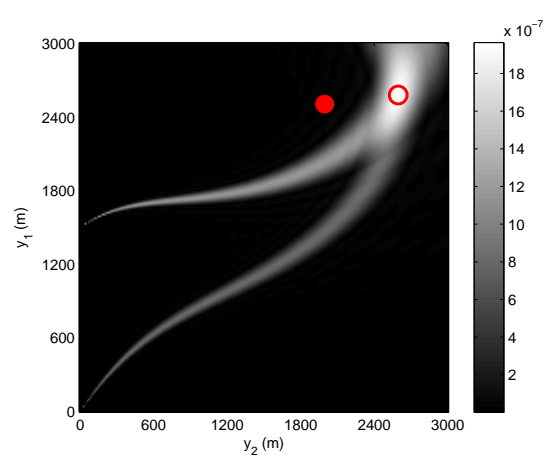

(b)

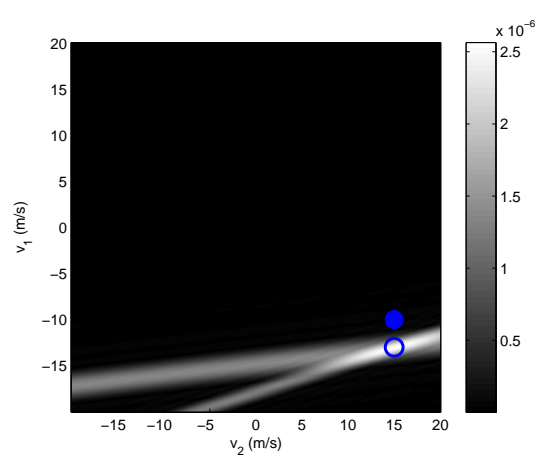

(c)

Figure 1: The reconstructed images for a moving point target with 3 receivers and a single transmitter transmitting a high-Doppler resolution waveform: (a) The peak-value image with the maximum value indicated by the circle. The estimated velocity $\tilde{\boldsymbol{v}}=(-13,15) \mathrm{m} / \mathrm{s}$. (b) The position-image when $\boldsymbol{v}=\tilde{\boldsymbol{v}}$. The estimated position $\tilde{\boldsymbol{y}}=(2580,2595) \mathrm{m}$. (c) The velocity-image when $\boldsymbol{y}=\tilde{\boldsymbol{y}}$. Solid dots indicate the true position (or velocity) and circles indicate the estimated position (or velocity).

Fig. 1(a) shows that, with 3 receivers, ambiguities are present in the peak-value image, indicated by the brightest (dark red) region in the image that spreads in the range of $[-16,-12] \mathrm{m} / \mathrm{s}$ in $\boldsymbol{v}_{1}$ and the entire range of $v_{2}$. Due to the strong ambiguities, the estimated velocity $\tilde{\boldsymbol{v}}=(-13,15) \mathrm{m} / \mathrm{s}$, deviates from the correct value as expected. The estimated position of the target, $(2580,2595) \mathrm{m}$, has error as well, as shown by the bright spot in Fig. 1(b). 


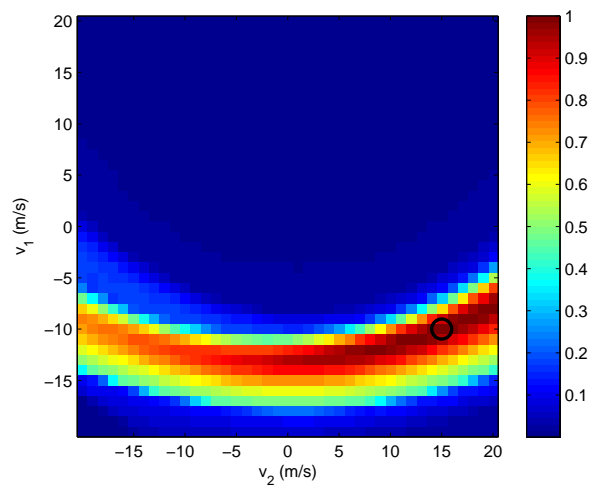

(a)

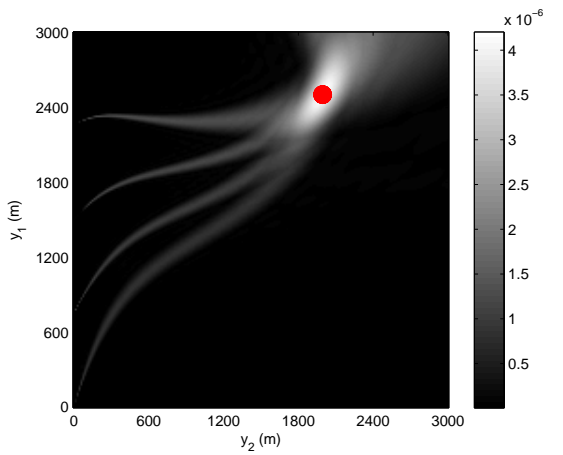

(b)

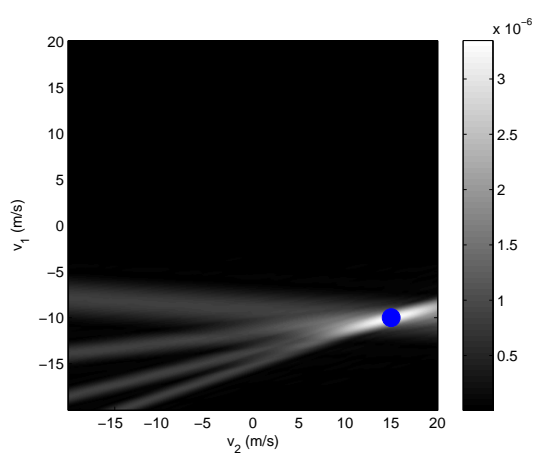

(c)

Figure 2: The reconstructed images for a moving point target with 5 receivers and a single transmitter transmitting a high-Doppler resolution waveform: (a) The peak-value image with the maximum value indicated by a circle. The estimated velocity $\tilde{\boldsymbol{v}}=(-10,15) \mathrm{m} / \mathrm{s}$. (b) The position-image when $\boldsymbol{v}=\tilde{\boldsymbol{v}}$. The estimated position $\tilde{\boldsymbol{y}}=(2500,2000) \mathrm{m}$. (c) The velocity-image when $\boldsymbol{y}=\tilde{\boldsymbol{y}}$. Solid dots indicate the true position (or velocity) and circles indicate the estimated position (or velocity).

The existence of the ambiguities is related to the velocity resolution of the imaging operator. As can be seen from Fig. 1(c), the velocity-image is ridge-like. Relatively strong ambiguities are present along the ridges. Comparing Fig. 1(c) with Fig. 1(a), we see that the ambiguities in the peak-value image are related to the ambiguities embedded in the velocity-image.

When the number of receivers increases, additional contours intersect at the correct target velocity, resulting in increased test-statistic value and thus, improved velocity resolution as shown in Fig. 2(c). Although the velocity-images are still ridge-like, the ambiguities along the ridges are much weaker as compared to that of the three-receiver case. This, in return, weakens the ambiguities in the peak-value image, as shown in Fig. 2(a).

Comparing the reconstructed position-images in Fig. 1(b) and Fig. 2(b), we see that similar to the reconstructed velocity-images, the strength of the position-image at the correct target location increases with the increasing number of receivers, which results in improved position resolution. In the five-receiver case, the target was reconstructed at the correct location, $(2500,2000) \mathrm{m}$, as shown in Fig. 2(a).

Fig. 3 shows the reconstruction results of a moving point target for the case of 5 receivers and 2 transmitters using the high-Doppler resolution waveform.

Comparing Fig. 3 with Fig. 2, which are the reconstructed results for the single-transmitter case, we see that in the multiple-transmitter case, the strength of the position image and velocity image at the target location and velocity increases with the increasing number of transmitters. However, multiple transmitters also induce artifacts in the reconstructed images. 


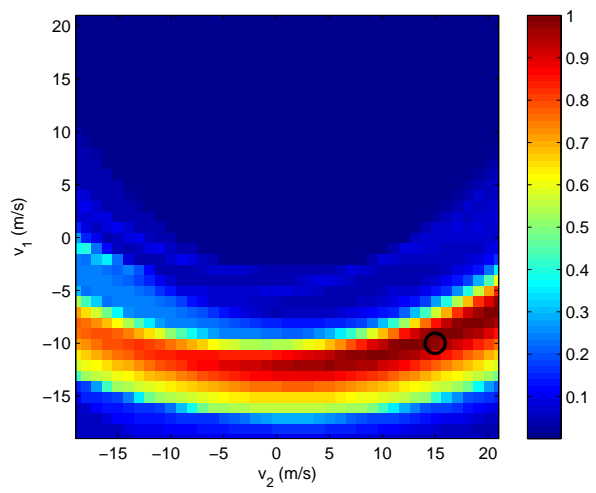

(a)

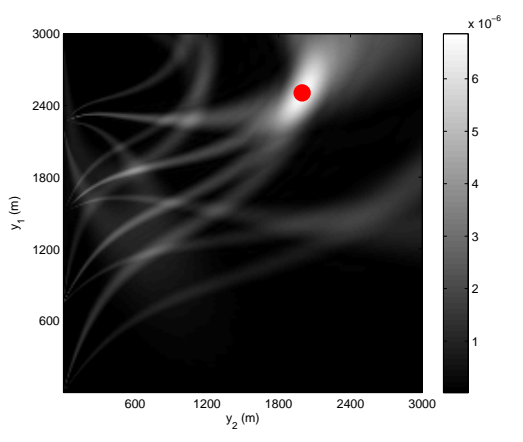

(b)

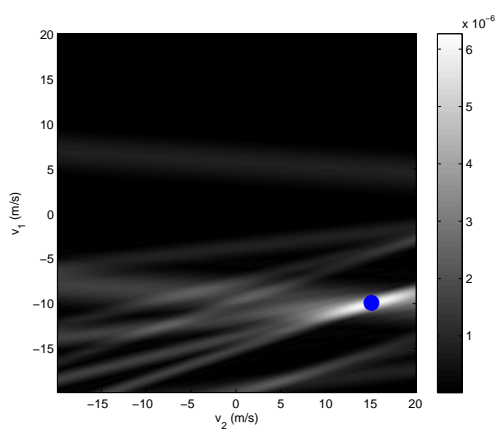

(c)

Figure 3: The reconstructed images for a moving point target with 5 receivers and 2 transmitters transmitting a high-Doppler resolution waveform: (a) The peak-value image with the maximum value indicated by a circle. The estimated velocity $\tilde{\boldsymbol{v}}=(-10,15) \mathrm{m} / \mathrm{s}$. (b) The position-image when $\boldsymbol{v}=\tilde{\boldsymbol{v}}$. The estimated position $\tilde{\boldsymbol{y}}=$ $(2500,2000) \mathrm{m}$. (c) The velocity-image when $\boldsymbol{y}=\tilde{\boldsymbol{y}}$. Solid dots indicate the true position (or velocity).

\section{CONCLUSION}

In this work, we presented a new passive imaging method for moving targets in position and velocity spaces using sparsely distributed receivers and non-cooperative transmitters of opportunity. We proposed a novel passive measurement model for moving targets. We formulated the passive image formation problem within a GLRT framework where we set up a test of binary hypothesis using the passive measurement model for a hypothetical target located at an unknown position, moving with an unknown velocity. We designed a linear discriminant functional by maximizing the SNR of the test-statistic. The resulting discriminant functional correlates the filtered, delayed and scaled (or dilated) averaged measurements from one receiver location with the measurements at another receiver location.

We presented numerical simulation results to demonstrate the performance of our passive imaging method. Resolution analysis of our imaging method will be the focus of our future work. We intend to perform theoretical and quantitative resolution analysis under a variety of scenarios. Additionally, our passive measurement model can be extended to be operating in multiple-scattering environments, which will be discussed in another paper.

Finally, we note that the passive moving target imaging method introduced in this paper is not limited to radar, and can be easily adapted to similar passive moving object imaging problems in acoustics, geophysics or microwave imaging. 


\section{REFERENCES}

[1] Griffiths, H. D. and Long, N. R. W., "Television-based bistatic radar," IEE Proceedings of Radar, Sonar and Navigation 133, 649-657 (July 1986).

[2] Griffiths, H. D. and Baker, C. J., "Passive coherent location radar systems. part 1: Performance prediction," IEE Proceedings of Radar, Sonar and Navigation 152, 153-159 (June 2005).

[3] Baker, C. J., Griffiths, H. D., and Papoutsis, I., "Passive coherent location radar systems. part 2: Waveform properties," IEE Proceedings of Radar, Sonar, and Navigation 152, 160-168 (June 2005).

[4] O'Hagan, D. W. and Baker, C. J., "Passive bistatic radar (PBR) using FM radio illuminators of opportunity," in [Proc. of 2008 IEEE Radar Conference, Roma, Italy], (May 2008).

[5] Koch, V. and Westphal, R., "New approach to a multistatic passive radar sensor for air/space defense," IEEE Aero. Electron. Syst. Mag. 10, 24-32 (Nov. 1995).

[6] Poullin, D., "Passive detection using digital broadcasters (DAB, DVB) with COFDM modulation," IEE Proceedings of Radar, Sonar and Navigation 152, 143-152 (June 2005).

[7] Tan, D. K. P., Sun, H., Lu, Y., Lesturgie, M., and Chan, H. L., "Passive radar using global system for mobile communication signal: theory, implementation and measurements," IEE Proceedings of Radar, Sonar, and Navigation 152, 116-123 (June 2005).

[8] Howland, P. E., Maksimiuk, D., and Reitsma, G., "Fm radio based bistatic radar," IEE Proceedings of Radar, Sonar and Navigation 152, 107-115 (June 2005).

[9] He, X., Cherniakov, M., and Zeng, T., "Signal detectability in SS-BSAR with GNSS non-cooperative transmitter," IEE Proceedings of Radar, Sonar and Navigation 152, 124-132 (June 2005).

[10] Kulpa, K. S., "Multi-static entirely passive detection of moving targets and its limitations," IEE Proceedings of Radar, Sonar, and Navigation 152, 169-173 (June 2005).

[11] Coleman, C. and Yardley, H., "Passive bistatic radar based on target illumniations by digital audio broadcasting," IET Radar Sonar Navig. 2(5), 366-375 (2008).

[12] Guo, H., Woodbridge, K., and Baker, C. J., "Evaluation of WiFi beacon transmissions for wireless based passive radar," in [Proc. of 2008 IEEE Radar Conference, Roma, Italy], (May 2008).

[13] Chetty, K., Woodbridge, K., Guo, H., and Smith, G. E., "Passive bistatic WiMAX radar for marine surveillance," in [Proc. of 2010 IEEE Radar Conference, Washington, DC, USA], (May 2010).

[14] Falcone, P., Colone, F., Bongioanni, C., and Lombardo, P., "Experimental results for OFDM WiFi-based passive bistatic radar," in [Proc. of 2010 IEEE Radar Conference, Washington, DC, USA], (May 2010).

[15] Harms, H. A., Davis, L. M., and Palmer, J., "Understanding the signal structure in DVB-T signals for passive radar detection," in [Proc. of 2010 IEEE Radar Conference, Washington, DC, USA], (May 2010).

[16] Christiansen, J. M. and Olsen, K. E., "Range and doppler walk in DVB-T based passive bistatic radar," in [Proc. of 2010 IEEE Radar Conference, Washington, DC, USA], (May 2010).

[17] Wu, Y. and Munson, D. C., "Multistatic synthetic aperture imaging of aircraft using reflecting television signals," in [Proc. of SPIE, Algorithms for Synthetic Aperture Radar Imagery VIII], (April 2001).

[18] Wu, Y. and Munson, D. C., "Wide-angle ISAR passive imaging using smoothed pseudo Wigner-Ville distribution," in [Proc. of 2001 IEEE Radar Conference], (May 2001).

[19] Kay, S., [Fundamentals of Statistical Signal Processing, Vol. I - Estimation Theory], Prentice Hall (1993).

[20] Kay, S., [Fundamentals of Statistical Signal Processing, Vol II - Detection Theory], Prentice Hall (1998).

[21] Colton, D. and Kress, R., [Inverse Acoustic and Electromagnetic Scattering Theory], vol. 93 of Applied Mathematical Sciences, Springer, 2 ed. (1998).

[22] Ghosh Roy, D. N. and Couchman, L. S., [Inverse Problems and Inverse Scattering of Plane Waves], Academic Press, London, UK (2002).

[23] Barrett, H. H. and Myers, K. J., [Foundations of Image Science], Wiley-Interscience (2003).

[24] Cheney, M. and Borden, B., "Imaging moving targets from scattered waves," Inverse Problems 24, 035005(122) (2008).

[25] Cheney, M., "A mathematical tutorial on synthetic aperture radar," SIAM Review 43(2), 301-312 (2001).

[26] Langenberg, K. J., Brandfass, M., Mayer, K., Kreutter, T., Brüll, A., Felinger, P., and Huo, D., "Principles of microwave imaging and inverse scattering," EARSeL Adv. Remote Sens. 2, 163-186 (1993). 
[27] Yarman, C. E., Wang, L., and Yazıcı, B., "Doppler synthetic aperture hitchhiker imaging," Inverse Problems 26 (065006) (2010).

[28] Wang, L., Son, I. Y., and Yazıcı, B., "Passive imaging using distributed apertures in multiple scattering environments," Inverse Problems 26 (065002) (2010). 